\title{
Fipronil upregulates inflammatory cytokines and MUC5AC expression in human nasal epithelial cells*
}

\author{
Soyoung Kwak' ${ }^{1,2}$, Yoon Seok Choi ${ }^{2}$, Hyung Gyun $\mathrm{Na}^{2}$, Chang Hoon Bae', \\ Si-Youn Song'2, Yong-Dae Kim²,3 \\ 'Department of Medical Science, College of Medicine, Graduate School of Yeungnam University, Daegu, Republic of Korea \\ 2 Department of Otorhinolaryngology-Head and Neck Surgery, College of Medicine, Yeungnam University, Daegu, Republic of \\ Korea \\ ${ }^{3}$ Regional Center for Respiratory Diseases, Yeungnam University Medical Center, Daegu, Republic of Korea
}

Rhinology 58: 1, $66-73,2020$

https://doi.org/10.4193/Rhin19.172

*Received for publication:

April 25, 2019

Accepted: August 25, 2019

\begin{abstract}
Background: Airway inflammation and excessive mucin production are pathophysiological characteristics of airway diseases. Fipronil, a pesticide, is being extensively used in agriculture and veterinary medicine worldwide. However, this compound impairs immune function in non-target organisms. The present study aimed to evaluate the effect of fipronil on pro-inflammatory cytokine and mucus production and signalling pathways in human primary nasal epithelial cells.
\end{abstract}

Methodology: The effect of fipronil on pro-inflammatory cytokine and MUC5AC expression and the signalling pathway of fipronil were investigated using real-time PCR, enzyme immunoassays, immunofluorescence, and immunoblot analysis with specific inhibitors and small interfering RNA.

Results: Fipronil treatment increased pro-inflammatory cytokine interleukin (IL)-1 $\beta$, IL-6, IL-8, and MUC5AC expression in human primary nasal epithelial cells. It also induced phosphorylation of extracellular signal-regulated kinase 1/2 (ERK1/2) mitogenactivated protein kinase (MAPK), p38 MAPK, and nuclear factor kappa-light-chain-enhancer of activated B cells (NF-KB). MAPK and NF- $\kappa B$ inhibitor treatment significantly inhibited increases in IL-1 $\beta$, IL-6, IL-8, and MUC5AC expression. Ex vivo data confirmed that fipronil-induced MUC5AC expression occurs through ERK1/2, p38, and NF-KB signalling pathways in nasal inferior turbinate tissue.

Conclusions: Fipronil induced pro-inflammatory cytokine IL-1 $\beta$, IL-6, IL-8, and MUC5AC expression via ERK1/2 MAPK, p38 MAPK, and NF-KB in human primary nasal epithelial cells.

Key words: Fipronil, mucus, MUC5AC, cytokines, human primary nasal epithelial cells

\section{Introduction}

The airway mucus layer plays a vital role as the first line of innate immune defence against invasion by microorganisms and damage to the underlying tissue ${ }^{(1)}$. Maintaining homeostasis in mucus production is an essential role of the innate immune system to ensure proper mucociliary clearance in normal airways ${ }^{(2)}$. Mucus is secreted in the upper and/or lower respiratory tracts during environmental challenges and in chronic infection conditions. Excessive mucus production is a characteristic of disease and it results in mucus accumulation, plugging of the airway lumen, recurrent airway infections, and inflammation (3). The major pathology of airway diseases, including asthma, chronic obstructive pulmonary disease (COPD), cystic fibrosis, and chronic rhinosinusitis, is characterized by obstruction of the airway lumen ${ }^{(4)}$. One of the causes of this obstruction is the presence of exudate containing mucus. Airway mucus is composed of water, ions, lipids, and various macromolecules. Mucins are high molecular-weight glycoproteins that it are essential to defend and protect the airway surface from inhaled toxicants and pathogens. Mucin is produced by epithelial goblet cells and submucosal glands. MUC5AC and MUC5B is a major gel-forming mucin that is linked to increased morbidity and mortality in respiratory diseases ${ }^{(5)}$. 
Pesticides are used in a wide variety of applications, including agricultural fields, grass, turf, and veterinary products. Fipronil is utilized as an attractive bait for ants and cockroaches, and in anti-flea and tick sprays to protect crops ${ }^{\left({ }^{6}\right)}$. This compound belongs to the phenylpyrazole insecticide family and it acts as an antagonist to GABA receptors. Fipronil exposure leads to uncontrolled blockage of glutamate-activated chloride channels in the central nervous system. It seems to be safe due to its selective toxicity toward many classes of insects and invertebrates. Although the use of the recommended dose of fipronil seems to be safe, prolonged or excessive fipronil use causes damage to non-target organisms, including animals and humans ${ }^{(7)}$. Previous studies reported that fipronil inhibits the electron transport chain in mitochondria, causing decreased ATP levels and inducing apoptosis ${ }^{(8,9)}$. Additionally, fipronil exposure significantly increases reactive oxygen species generation and peroxidation as markers of oxidative stress in the kidneys and brains of mice ${ }^{(10)}$. In addition, it causes lung inflammation and monocyte cell death upon oral and intranasal administration ${ }^{(11)}$. However, there are no data regarding how fipronil affects levels of proinflammatory cytokines and major airway mucin overproduction in human primary nasal epithelial cells. Therefore, this study was designed to investigate the airway inflammation of fipronil through testing whether it affects pro-inflammatory cytokine levels and major mucin overproduction in human primary nasal epithelial cells.

\section{Materials and methods}

\section{Materials}

Fipronil was obtained from Sigma-Aldrich (St. Louis, MO, USA). Commercial human primary airway epithelial cells of nasal origin (hAEC) were purchased from Epithelix (EP40, donor information: 14/male/caucasian, nasal mucosa, freezing medium, Geneva, Switzerland). The specific inhibitors U0126, SB203580, and BAY 11-7085 were purchased from Calbiochem (San Diego, CA, USA), BIOMOL (Plymouth Meeting, PA, USA), and Sigma-Aldrich, respectively. MUC5AC primer (QT01329615, Qiagen, Hilden, Germary) and MUC5B primer (BioRad 10025636, qHsaCIP0028135, Hercules, CA, USA) were purchased. For transfection with small interfering RNA (siRNA), predesigned siRNA (extracellular signalregulated kinase ERK1, ERK2, and p38) were obtained. control siRNA (\#6568) and nuclear factor kappa-light-chain-enhancer of activated B cells, NF-KB siRNA (\#6261) were purchased from Cell signalling technology.

\section{Tissue collection}

Nasal inferior turbinate tissues from 10 patients undergoing augmentation rhinoplasty and inferior turbinoplasty for correction of cosmetic problems (females, mean age: 40.2 years, non-smoker) in patients without rhinitis/rhinosinusitis. They had no personal or family history of allergy and negative results on both allergy specific tests (skin-prick test and multiple allergen simultaneous test). This study was approved by the Institutional Review Board for human studies at the Yeungnam University Medical Center, and written informed consents were obtained from all patients (YUMC 2018-09-033).

\section{Cell culture and treatment}

Human nasal epithelial cells were cultured in airway epithelial cell growth medium at $37^{\circ} \mathrm{C}$ in $5 \% \mathrm{CO}_{2}$-fully humidified air, and subcultured according to the manufacturer's instructions. To investigate the signalling pathways involved in the expression of pro-inflammatory cytokines IL-1 $\beta$, IL-6, IL-8, and MUC5AC, cultured cells were pre-treated with a specific inhibitor for $1 \mathrm{~h}$ before exposure to fipronil, whereas control cells were incubated with the culture medium for $1 \mathrm{~h}$.

\section{Cell viability assay}

Water-soluble tetrazolium salt-1 (WST-1) was used as an indicator of cell viability and proliferation. Cells were grown in 96-well plates at a density of $1 \times 10^{4}$ cells/well. After $24 \mathrm{~h}$, cells were washed with fresh medium and treated with fipronil or culture medium as a control. After incubation for $48 \mathrm{~h}$, cells were washed and $10 \mu \mathrm{L}$ of EZ-Cytox cell viability assay reagent (Daeil Lab, Seoul, Korea) was added, followed by incubation for $4 \mathrm{~h}$. The amount of formazan salt was determined by measuring the absorbance at $450 \mathrm{~nm}$ (reference wavelength 600-650 nm) using a microplate reader (Tecan Austria $\mathrm{GmbH}$, Austria).

\section{Real-time PCR analysis}

To investigate the effects of fipronil on pro-inflammatory cytokine IL-1 $\beta$, IL-6, IL-8, and MUC5AC expression in human primary nasal epithelial cells were treated with different concentrations of fipronil for $8 \mathrm{~h}$. The primers used were as follows: IL-1 $\beta, 5^{\prime}$ ATG CAC CTG TAC GAT CAC TG-3' (forward), 5'-ACA AAG GAC ATG GAG AAC ACC-3' (reverse); IL-6, 5'-GGTACATCCTCGACGGCATCT-3' (forward), 5'-GTG CCT CTT TGC TGC TTT CAC-3' (reverse); IL-8, 5'-ATG ACT TCC AAG CTG GCC GTG GCT-3' (forward), 5'-TCT CAG CCC TCT TCA AAA ACT TCT C-3' (reverse). Real-time $P C R$ was performed using the iQ SYBR Green Supermix (Bio-Rad, Hercules, CA, USA), according to the manufacturer's protocol. Briefly, $1 \mathrm{ng} / \mu \mathrm{L}$ of total RNA was reverse transcribed. A maximum of $2 \mu \mathrm{L}$ of each cDNA sample was used per $20 \mu \mathrm{L}$ of PCR mixture. $P C R$ reactions were carried out using a CFX96 real-time $P C R$ system C1000 thermal cycler (Bio-Rad, Hercules, CA, USA) up to triplicate wells and 40 cycles.

\section{Enzyme-linked immunosorbent assay (ELISA)}

Cell supernatants were collected from human nasal epithelial cells. Samples were diluted with PBS, transferred to a F96 Cert. Maxisorp Nunc-Immuno plate (Fisher scientific, Lenexa, KS, USA), and incubated at $4{ }^{\circ} \mathrm{C}$ overnight. Subsequently, samples 
were blocked with $2 \%$ bovine serum albumin (BSA) for $1 \mathrm{~h}$ and incubated with the following primary antibodies: rabbit anti-MUC5AC (H-160) (sc-20118; Santa Cruz Biotechnology, USA; 1:200 dilution), IL-1 $\beta$ (\#12242; Cell Signaling Technology, USA; 1:1000 dilution), IL-6 (ab6672; Abcam, Cambridge, UK; 1:1000 dilution), and IL-8 (M801; Thermo Scientific, IL, USA; 1:1000 dilution) in PBS containing $0.05 \%$ Tween 20 for $1 \mathrm{~h}$. Then, they were incubated with a horseradish peroxidase (HRP)-conjugated secondary antibody. After $1 \mathrm{~h}$, colour was developed using 3,3'5,5'-tetramethylbenzidine peroxidase solution, and the reaction was stopped using $2 \mathrm{~N} \mathrm{H}_{2} \mathrm{SO}_{4}$. Optical density was measured using an EL800 ELISA reader at $450 \mathrm{~nm}$ (BIO-TEK Instruments, Winooski, VT, USA). Results were expressed as fold increase from the baseline control.

\section{Western blot analysis}

Western blot analyses were performed to investigate the activation of MAPKs. Human nasal epithelial cells were seeded in 6-well plates and treated with the indicated concentrations of fipronil. Cells were harvested in $200 \mu \mathrm{L}$ of radioimmunoprecipitation assay buffer (Thermo Scientific, Rockford, IL, USA) with phosphatase inhibitor cocktail (Roche, Mannheim, Germany) and incubated for $20 \mathrm{~min}$ at $4{ }^{\circ} \mathrm{C}$. Subsequently, the samples were centrifuged at $2,500 \times \mathrm{g}$ for $10 \mathrm{~min}$ at $4{ }^{\circ} \mathrm{C}$, and the supernatant (whole-cell lysate) was collected. Proteins $(20 \mu \mathrm{g})$ were separated using $10 \%$ reducing sodium dodecyl sulphate-polyacrylamide gel electrophoresis and transferred onto a polyvinylidene difluoride membrane. The membrane was blocked with $5 \%$ BSA in Tris-buffered saline with $0.1 \%$ Tween 20 (TBST) and incubated with the following primary antibodies: rabbit antipERK1/2 (T202/Y204) (\#4370; Cell Signaling Technology, USA; 1:1000 dilution), rabbit anti-ERK1/2 (\#9102; Cell Signaling Technology, USA; 1:1000 dilution), rabbit anti-phospho-p38 (T180/ Y182) (\#9211; Cell Signaling Technology, USA; 1:1000 dilution), rabbit anti-p38 (H-147) (sc-7149; Santa Cruz Biotechnology, USA; 1:1000 dilution), rabbit anti-phospho-NF-KB p65 (Ser536) (93H1) (\#3033; Cell Signaling Technology, USA; 1:1000 dilution), and rabbit anti-NF-kB p65 (C22B4) (\#4764; Cell Signaling Technology, USA; $1: 1000$ dilution), at $4^{\circ} \mathrm{C}$ for $24 \mathrm{~h}$. After washing with TBST, blots were incubated with a secondary antibody (goat anti-rabbit lgG-HRP; sc 2004; Santa Cruz Biotechnology, USA; 1:1000 dilution) for $2 \mathrm{~h}$. Signals corresponding to the proteins of interest were developed using an enhanced West Pico chemiluminescent substrate (Thermo Fisher, Boston, MA, USA). Bands were detected after exposing the membrane to an imaging system (Chemiluminescence image system, FUSION-FX7 820wL, Eberhardzell, Germany).

\section{Cell transfections with siRNAs}

We examined whether ERK1/2, p38, and NF-KB signalling pathways were involved in fipronil-induced MUC5AC expres- sion in human primary nasal epithelial cells by pre-treating the cells with MAPK inhibitors and transfecting with siRNAs. The primer sequences used in the PCR were 5'- GCA AUG ACC AUA UCU GCU ACU UCC U-3' (forward), 5'-AGG AAG UAG CAG AUA UGG UCA UUG C-3' (reverse) for ERK1 siRNA, 5'-CCG AAG CAC CAU UCA AGU UCG ACA U-3' (forward), 5'- AUG UCG AAC UUG AAU GGU GCU UCG G-3' (reverse) for ERK2 siRNA, 5'- CCA AAU UCU CCG AGG UCU AAA GUA U-3' (forward), 5' - AUA CUU UAG ACC UCG GAG AAU UUG G-3' (reverse) for p38 siRNA. Human primary nasal epithelial cells were seeded in 6-well plates until they reached $70-80 \%$ confluence. Subsequently, the culture medium was changed to OPTI-MEM I reduced serum medium (Life Technologies, Carlsbad, CA, USA). siRNA and Lipofectamine 2000 (Life Technologies, Carlsbad, CA, USA) were pre-incubated together in OPTI-MEM I reduced serum medium for $20 \mathrm{~min}$ to form siRNA-Lipofectamine complexes, which was added to each well at a final siRNA concentration of $100 \mathrm{nM}$. Transfected cells were incubated for $48 \mathrm{~h}$ at $37^{\circ} \mathrm{C}$ in a $\mathrm{CO}_{2}$ incubator and then exposed to the indicated concentrations of fipronil. The transfection efficiency was $>90 \%$.

Ex vivo tissue culture and Immunofluorescence staining To confirm the signalling pathway involved in fipronil-induced MUC5AC expression in human nasal inferior turbinate tissue, we performed immunofluorescence (IF) staining in ex vivo culture. Tissues were cut into 2 - to $3-\mathrm{mm}^{3}$ pieces under sterile conditions. Before proceeding with the culture, the tissues were washed 3 times with phosphate buffered saline (PBS). After 24 $\mathrm{h}$ of fipronil $(10 \mu \mathrm{M})$ treatment, tissue fragments were fixed in $4 \%$ paraformaldehyde (PFA) in PBS for $24 \mathrm{~h}$, after which tissues were washed with PBS. Fixed tissues were embedded with optimal cutting temperature (OCT) compound for $1 \mathrm{~h}$ at $-80^{\circ} \mathrm{C}$. IF analyses were performed on 6- $\mu \mathrm{m}$ thick sections and blocked in $1 \%$ BSA in PBST for $1 \mathrm{~h}$ at room temperature. The slides were incubated with a rabbit anti-MUC5AC antibody (ab-198294; Abcam, Cambridge, UK; 1:100 dilution). Subsequently, they were incubated with an Alexa 488-labelled goat anti-rabbit lgG secondary antibody (green fluorescence). Nuclei were stained with 4,6-diamidino-2-phenylindole (DAPI) (blue fluorescence; Abcam, Cambridge, UK). The cells were visualized by fluorescence microscopy using Nikon software (Ti-S, 733551, Nikon, Tokyo, Japan) at $200 \times$ magnification.

\section{Statistical analysis}

Statistical analyses were performed using SPSS, version 12.0 software (SPSS, Chicago, IL, USA). Data were expressed as the mean \pm standard deviation (SD). Comparisons were performed using the unpaired t-test or Kruskal-Wallis test followed by a Mann-Whitney test. For all tests, a $p$ value $<0.05$ was considered statistically significant. 
A

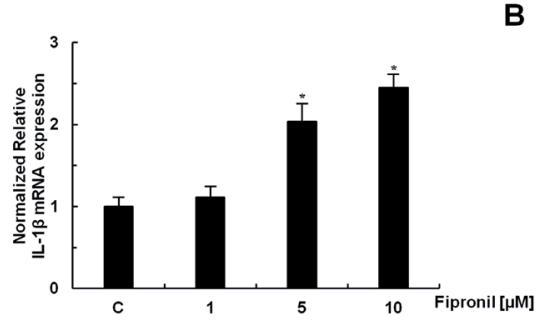

E

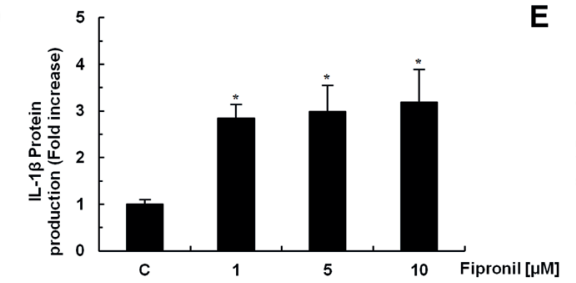

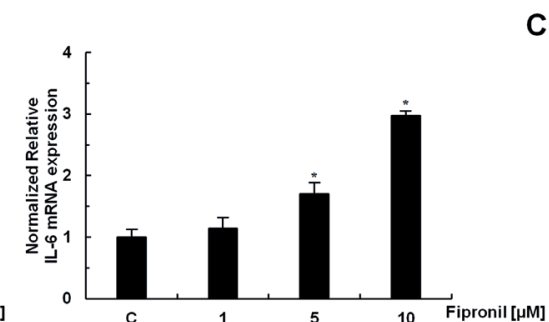

C

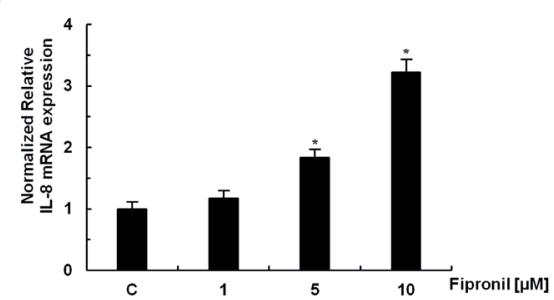

$\mathbf{F}$

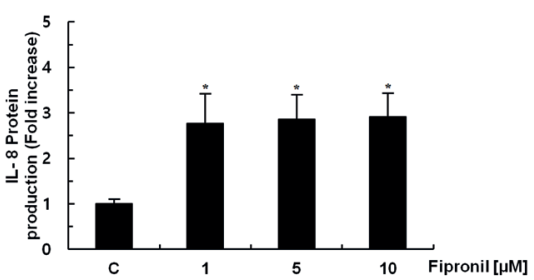

Figure 1. Effects of fipronil on levels of pro-inflammatory cytokines IL-1 $\beta$, IL-6, and IL-8 expression in human primary nasal epithelial cells. (A-C) Realtime PCR results showed that mRNA levels of IL-1 $\beta$, IL-6, and IL-8 were significantly and concentration-dependently increased upon exposure to fipronil. (D-F) ELISA results showed that IL-1 $\beta$, IL-6, and IL-8 protein production was increased by fipronil treatment. Figure shows a representative of three separate experiments performed in triplicate. Bars indicate the means \pm S.D. of three independent experiments performed in triplicate. C, control; ELISA, enzyme-linked immunosorbent assay; GAPDH, glyceraldehyde 3-phosphate dehydrogenase; real-time PCR, real-time polymerase chain reaction. ${ }^{*} p<0.05$ versus the control group.

A

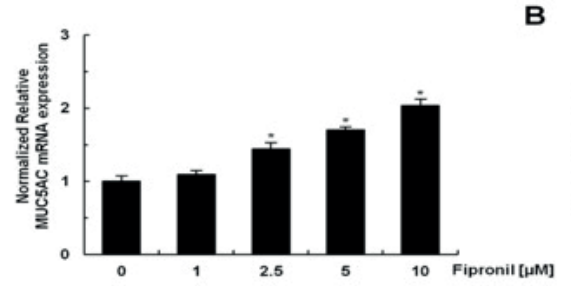

c

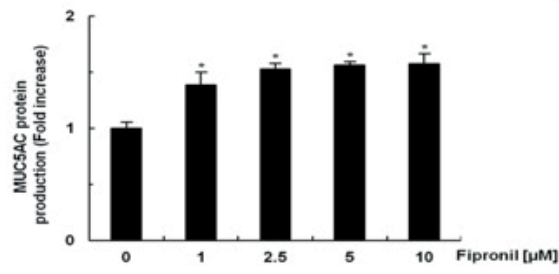

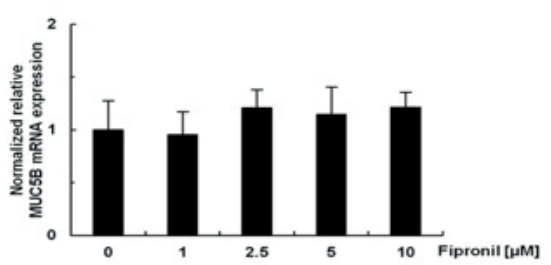

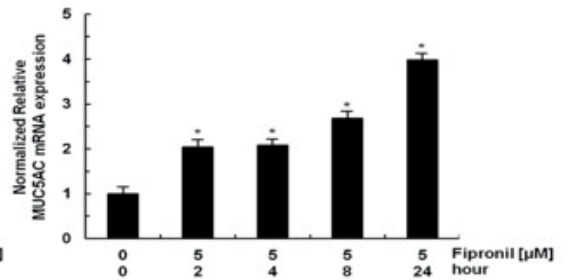

Figure 2. Effects of fipronil on levels of MUC5AC expression in human primary nasal epithelial cells. (A) Real-time PCR results showed that mRNA levels of MUC5AC significantly and concentration-dependently increased upon exposure to fipronil. (B) MUC5B mRNA expression was not induced by fipronil. (C) ELISA results showed that MUC5AC protein production was increased by fipronil treatment. (D) MUC5AC mRNA expression peaked $24 \mathrm{~h}$ after exposure to fipronil. Figure shows a representative of three separate experiments performed in triplicate. Bars indicate the means \pm S.D. of three independent experiments performed in triplicate. ELISA, enzyme-linked immunosorbent assay; GAPDH, glyceraldehyde 3-phosphate dehydrogenase; real-time $P C R$, real-time polymerase chain reaction. ${ }^{*} p<0.05$ versus the zero value.

\section{Results}

Fipronil induced inflammatory cytokine expression in human primary nasal epithelial cells

Fipronil did not affect cell viability at concentrations up to $20 \mu \mathrm{M}$ in human primary nasal epithelial cells (data not shown). Fipronil induced IL-1 $\beta$, IL-6, and IL-8 mRNA expression as shown by realtime PCR (Figures $1 \mathrm{~A}-\mathrm{C}$ ). A significant increase was detected at a fipronil concentration of $10 \mu \mathrm{M}$. ELISA showed that IL-1 $\beta$, IL-6, and IL-8 protein levels were significantly increased upon fipronil treatment at different concentrations (Figures 1D-F).

\section{Fipronil induced MUC5AC expression in human primary nasal epithelial cells}

Real-time PCR and ELISA showed that Fipronil induced MUC5AC expression at different concentrations (Figures $2 \mathrm{~A}$ and $\mathrm{C}$ ). However, MUC5B expression was not induced by fipronil (Figure 2B). 
A
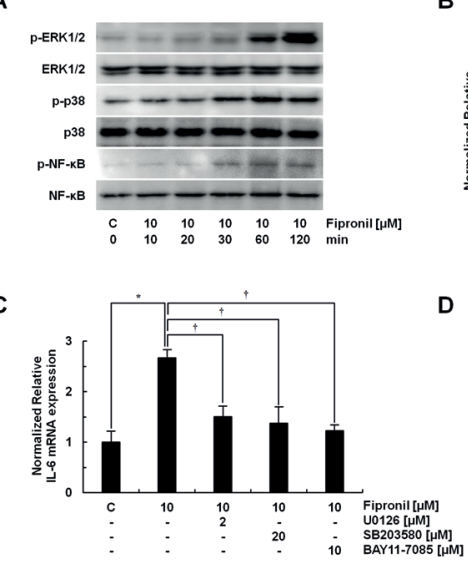

D
B

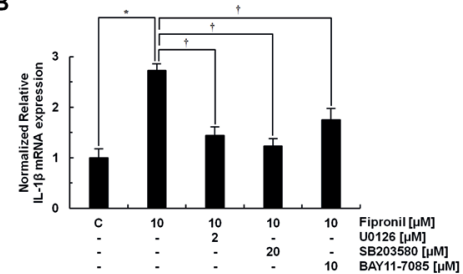

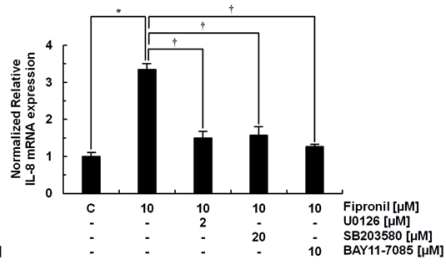

Figure 3. Fipronil-induced pro-inflammatory cytokine IL-1 $\beta$, IL-6, and IL-8 expression in human primary nasal epithelial cells was associated with ERK1/2, p38, and NF-KB activation. (A) Results of Western blot analyses showed that fipronil treatment induced the phosphorylation of ERK1/2, p38, and NF-KB. (B-D) Results of real-time PCR showed that U0126 (ERK1/2 inhibitor), SB203580 (p38 inhibitor), and BAY 11-7085 (NF-KB inhibitor) significantly attenuated fipronil-induced IL-1 $\beta$, IL-6, and IL-8 mRNA expression. Figure shows a representative of three separate experiments performed in triplicate. Bars indicate the means \pm S.D. of three independent experiments performed in triplicate. C, control; ELISA, enzyme-linked immunosorbent assay; ERK, extracellular signal-regulated kinase; GAPDH, glyceraldehyde 3-phosphate dehydrogenase; NF-KB, nuclear factor kappa-light-chainenhancer of activated $B$ cells; $p$-ERK MAPK, phosphorylated ERK MAPK; p-NF-kB, phosphorylated NF-kB; p-p38 MAPK, phosphorylated p38 MAPK. *p $<0.05$ versus the control group, $+p<0.05$ versus fipronil $(10 \mu \mathrm{M})$ alone.

A
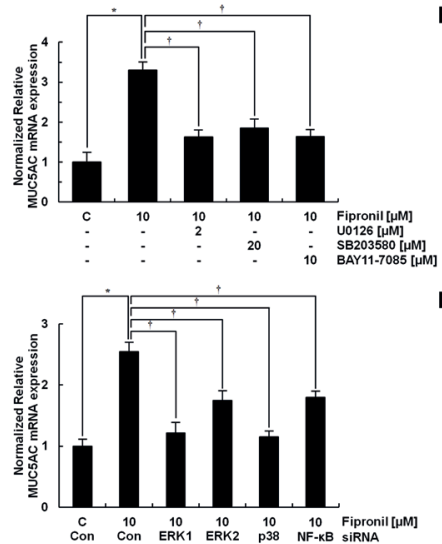

B
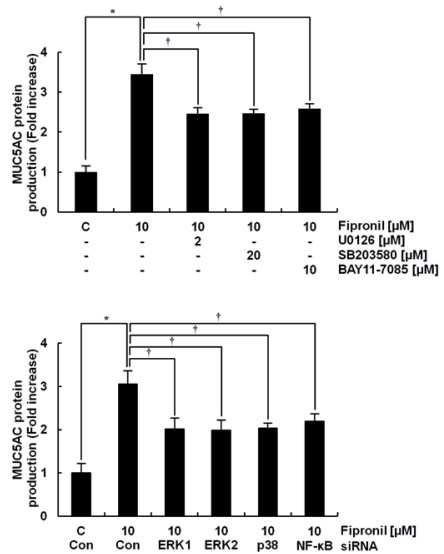

Figure 4. Fipronil-induced MUC5AC expression in human primary nasal epithelial cells was associated with ERK1/2, p38, and NF-KB activation. (A and B) Results of real-time PCR and ELISA showed that U0126 (ERK1/2 inhibitor), SB203580 (p38 inhibitor), and BAY 11-7085 (NF-KB inhibitor) significantly attenuated fipronil-induced MUC5AC mRNA expression and protein production. (C and D) Results of real-time PCR and ELISA showed that knockdown of ERK1, ERK2, p38, and NF-KB by siRNA significantly blocked fipronil-induced MUC5AC mRNA expression and protein production. Figure shows a representative of three separate experiments performed in triplicate. Bars indicate the means \pm S.D. of three independent experiments performed in triplicate. C, control; Con, control siRNA; ELISA, enzyme-linked immunosorbent assay; ERK, extracellular signal-regulated kinase; GAPDH, glyceraldehyde 3-phosphate dehydrogenase; NF-KB, nuclear factor kappa-light-chain-enhancer of activated B cells; $\mathrm{P}$-ERK MAPK, phosphorylated ERK MAPK; p-NF-KB, phosphorylated NF-KB; p-p38 MAPK, phosphorylated p38 MAPK. ${ }^{*} \mathrm{p}<0.05$ versus the contorl group, $\mathrm{tp}<0.05$ versus fipronil (10 $\left.\mu \mathrm{M}\right)$ alone.

Additionally, fipronil effect began from $2 \mathrm{~h}$ after incubation and reached its maximum at $24 \mathrm{~h}$ (Figure $2 \mathrm{D}$ ).

Fipronil-induced cytokine expression in human primary nasal epithelial cells was associated with phosphorylation of

\section{ERK1/2, p38, and NF-KB}

Fipronil treatment resulted in the phosphorylation (activation) of ERK1/2, p38, and NF-KB (Figure 3A). Furthermore, we examined whether ERK1/2, p38, and NF-KB signalling pathways were involved in fipronil-induced pro-inflammatory cytokine 


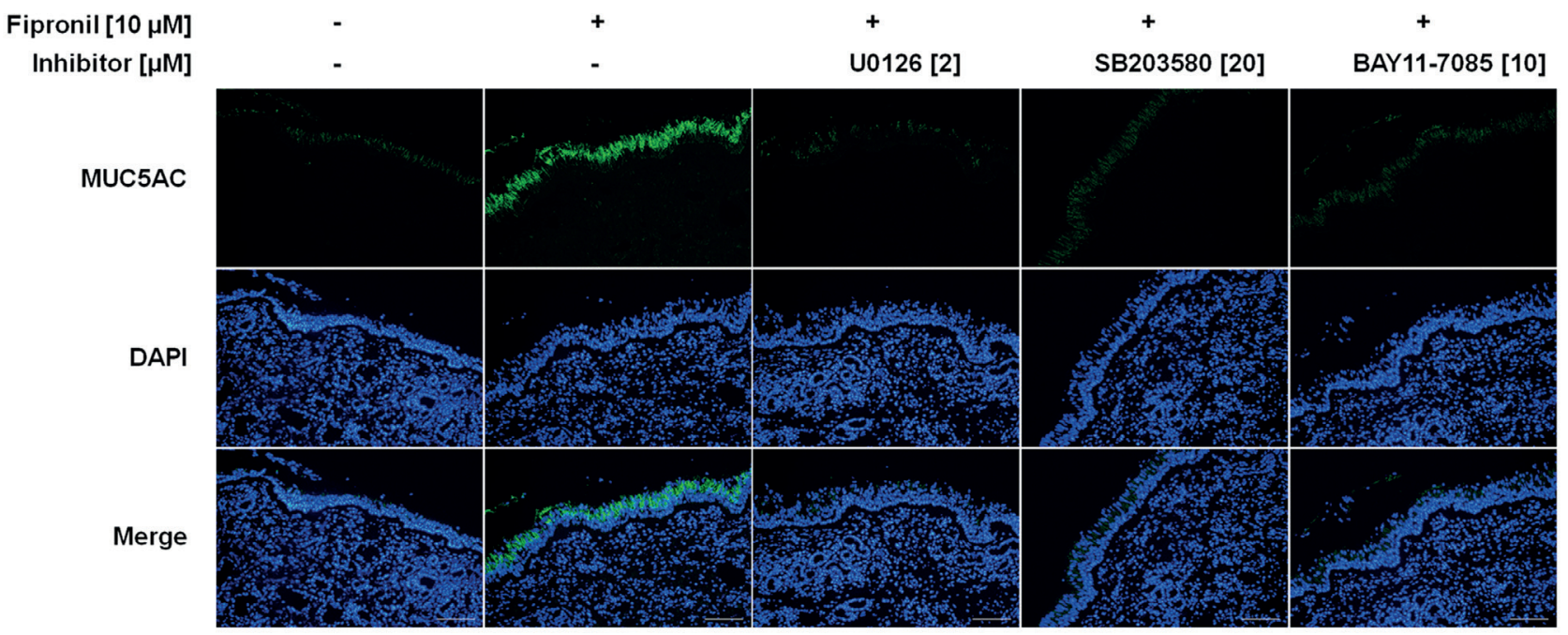

Figure 5. Fipronil-induced MUC5AC protein production in human nasal inferior turbinate tissues was associated with ERK1/2, p38, and NF-KB activation. Representative immunofluorescence staining showed MUC5AC (green) with nuclear DAPI (blue). Fipronil significantly induced MUC5AC protein production (green) in the epithelium. Treatment with a specific ERK1/2 inhibitor (U0126), p38 inhibitor (SB203580), and NF-KB inhibitor (BAY 11-7085) significantly attenuated fipronil-induced MUC5AC protein production (green). Ex vivo cultures from different donors were performed at least three independent experiments. DAPI, 4',6-diamidino-2-phenylindole. Scale bars, $100 \mu \mathrm{m}$.

IL-1 $\beta$, IL-6, IL-8 expression in human primary nasal epithelial cells by pre-treating the cells with MAPK inhibitors. Results of real-time PCR showed that U0126 (a specific ERK1/2 inhibitor), SB203580 (a specific p38 inhibitor), and BAY 11-7085 (a NF-KB inhibitor) significantly attenuated fipronil-induced pro-inflammatory cytokine IL-1 $\beta$, IL-6, IL-8 expression (Figures 3B-D).

\section{Fipronil-induced MUC5AC expression in human primary} nasal epithelial cells was associated with ERK1/2, p38, and NF-KB activation

Results of real-time PCR and ELISA showed that treatment with U0126, SB203580, and BAY 11-7085 significantly attenuated fipronil-induced MUC5AC mRNA expression and protein production (Figures 4A and B). Knockdown of ERK1, ERK2, p38, and NF$\mathrm{KB}$ by siRNA significantly attenuated fipronil-induced MUC5AC mRNA expression (Figures $4 C$ and D).

Fipronil-induced MUC5AC expression was associated with ERK1/2, p38, and NF-KB in tissue culture of human nasal inferior turbinate tissue

Results of IF showed that treatment with the MAPK inhibitors (U0126 and SB2035800) and NF-KB inhibitor (BAY 11-7085) significantly attenuated fipronil-induced MUC5AC protein production in the epithelium (Figure 5).

\section{Discussion}

Fipronil, a pesticide, has a long half-life that ranges from 3-3.7 months in the field, depending on factors such as soil type, ultraviolet radiation, moisture, and temperature ${ }^{(12)}$. It is easy to contaminate cow milk, fruit, and vegetables with pesticides. Notably, millions of eggs have been found with excessive fipronil levels in Europe and South Korea in 2017. Fipronil can bioaccumulate through a variety of methods including residue intake via food, inhalation, and dermal acute exposure. This compound is readily metabolized and slowly excreted via urine, faeces, and bile ${ }^{(13)}$. The major metabolite of fipronil in humans is fipronil sulfone, which is also harmful to human health ${ }^{(14)}$. Fipronil metabolites have been shown to persist longer than fipronil itself. In this study, we observed that fipronil treatment induced the expression of pro-inflammatory cytokines IL-1 $\beta$, IL-6, and IL-8, and MUC5AC expression via ERK1/2, p38, and NF-KB signalling pathways in human primary nasal epithelial cells and human nasal inferior turbinate tissue. Fipronil also activated ERK1/2, p38, and NF-KB signalling pathways. Inhibitors and siRNA of ERK1/2, p38, and NF-KB inhibited fipronil-induced cytokines and MUC5AC expression.

Pro-inflammatory cytokines, including IL-1 $\beta$, IL- 6 and IL-8, play important roles in the immune and inflammatory responses $(15,16)$. These cytokines are implicated in many airway inflammatory diseases as general pro-inflammatory markers and are used to determine whether it relate in response to airway inflammation. IL-1 $\beta$ and IL- 6 are produced not only innate immune cells including macrophages, dendritic cells, mast cells, neutrophils, and $B$ cells but also non-leukocytes such as fibroblasts, endothelial cells, and epithelial cells. IL-1 $\beta$ and IL- 6 are frequently found in sputum and bronchoalveolar lavage fluid of patients with asthma and COPD and amplify inflammation through the activation of NF-KB. IL-8, a neutrophil attractant and activator, plays 
a significant role in airway inflammation ${ }^{(17)}$. In a recent study, bronchial epithelial cells from patients with COPD exhibited increased levels of IL-8 ${ }^{(18)}$. Fipronil may aggravate airway inflammatory diseases that are related with increased pro-inflammatory cytokine such as IL-1 $\beta$, IL- 6 and IL-8. We demonstrated that fipronil significantly increased the expression of pro-inflammatory cytokines such as IL-1 $\beta$, IL-6, and IL-8 (Figure 2).

Mucus covers the luminal surfaces of epithelial organs and protects the respiratory tract by trapping inhaled irritants and pathogens. Goblet cells and submucosal glands produce mucin, which determines viscoelasticity. During airway infection or trauma, epithelial cells lining the airway tract undergo goblet cell metaplasia and hyperplasia related to activation of the MAPK/NF-KB signalling pathway. MUC5AC, a representative secretory-associated mucin, is a goblet cell marker. Goblet cell differentiation and excessive mucus production are common features of many airway diseases ${ }^{(19)}$. Patients with COPD and asthma exhibit increased goblet cell hyperplasia and luminal mucin production ${ }^{(20-22)}$. MUC5AC levels are increased by goblet cell hyperplasia and metaplasia in sinus mucosa of patients with chronic rhinosinusitis ${ }^{(23,24)}$.

Results of this study showed that fipronil significantly increased MUC5AC expression in human primary nasal epithelial cells (Figures 2A and C). However, fipronil did not induce MUC5B expression. We think that the reason of the different expression between MUC5AC and MUC5B are morphological and biophysical properties. MUC5B is related to control of infection in upper airway and maintain immune homeostasis in lower airway whereas MUC5AC had not influence ${ }^{(25)}$. Moreover, two mucins suggest different localization that the MUC5AC is normally produced in epithelium by goblet cells and MUC5B is mainly produced submucosal glands. A recent study, these are verified different morphology that MUC5B is liner form like bundles coated by MUC5AC ${ }^{(26)}$. Thus, present study, it was expected the expression of two mucins would be regulated different ways. Expression of pro-inflammatory cytokines and MUC5AC was induced through ERK1/2, p38, and NF-KB activation by various inflammatory mediators in human airway epithelial cells. MAPKs, serine-threonine protein kinases, play a critical role in various airway inflammatory signals. Treatment with a MAPK activity inhibitor can block airway inflammatory disease. Moreover, NF-KB signalling regulates cytokine activity in airway pathology. Therefore, we focused on the role of signalling associated with ERK1/2, p38, and NF-KB in fipronil-induced expression of proinflammatory cytokines IL-1 $\beta$, IL-6, IL-8, and MUC5AC. Fipronil treatment induced the phosphorylation of ERK1/2, p38, and NF-KB. MAPK-specific inhibitors significantly attenuated fipronilinduced IL-1 $\beta$, IL-6, IL-8 and MUC5AC expression and phosphorylation of ERK1/2, p38, and NF-KB. Ex vivo data confirmed that fipronil-induced MUC5AC was associated with ERK1/2-p38 and NF-kB signalling pathways in nasal inferior turbinate tissue.
These findings suggested that fipronil induced upper airway inflammation and MUC5AC expression in human primary nasal epithelial cells through ERK1/2, p38, and NF-KB. However, there are some limitations in this study. Currently, the treatment of airway diseases with mucin production and inflammatory responses have used corticosteroid and anti-inflammatory agent. However, we just focus on effect of fipronil on mucin production and inflammation responses in nasal epithelial cells. Further studies are necessary to clarify effect of therapeutic agent such as corticosteroid and anti-inflammatory agent on mucin production and inflammatory responses in nasal epithelial cells ${ }^{(27)}$. And the present study did not demonstrate the potential receptor(s) bind to fipronil. We will do further experiment to elucidate which receptor regulates activation of these signaling pathway and the more precise regulatory mechanisms. And, we will also compare the effect of fipronil and other inducers such as lipopolysaccharide on mucin production and inflammatory response and determine synergic effect with fipronil and other inducer.

\section{Conclusion}

In conclusion, this study showed, for the first time, that fipronil exposure induced pro-inflammatory cytokine IL-1 $\beta$, IL-6, IL-8, and MUC5AC expression via activation of ERK1/2-p38 and NF-KB pathways in human primary nasal epithelial cells and nasal inferior turbinate tissue. Although additional studies are necessary to fully understand the effects of fipronil on the expression of cytokines and mucin and the detailed signalling pathways involved, these findings provide important insights into the ability of fipronil to regulate cytokine and mucin secretion in upper airway epithelial cells.

\section{Acknowledgements}

This work was supported by the 2017 Yeungnam University Research Grant.

\section{Authorship contribution}

SYK: experimentation;

YSC: study design;

HGN: data collection, writing;

CHB: manuscript revision;

SYS: data analysis, data interpretation;

YDK: study concept, supervision.

\section{Conflict of interest}

The authors state that there is no conflict of interest regarding this manuscript. 


\section{References}

1. Ma J, Rubin BK, Voynow JA. Mucins, Mucus, and Goblet Cells. Chest, 2018; 154: 169-176.

2. Martinez-Anton A, de Bolos C, Garrido M, Roca-Ferrer J, Barranco C, Alobid I, et al. Mucin genes have different expression patterns in healthy and diseased upper airway mucosa. Clin Exp Allergy, 2006; 36: 448-457.

3. Turner J, Jones CE. Regulation of mucin expression in respiratory diseases. Biochem Soc Transact, 2009; 37: 877-881.

4. Ali MS, Pearson JP. Upper airway mucin gene expression: a review. Laryngoscope, 2007; 117: 932-938.

5. Gray T, Coakley R, Hirsh A, Thornton D, Kirkham S, Koo JS, et al. Regulation of MUC5AC mucin secretion and airway surface liquid metabolism by IL-1beta in human bronchial epithelia. Am J Physiol Lung Cell Mol Physiol, 2004; 286: L320-330.

6. Gibbons D, Morrissey C, Mineau P. A review of the direct and indirect effects of neonicotinoids and fipronil on vertebrate wildlife. Environ Sci Pollut Res Int, 2015; 22: 103-118.

7. Hayasaka D, Kuwayama N, Takeo A, Ishida T, Mano H, Inoue MN, et al. Different acute toxicity of fipronil baits on invasive Linepithema humile supercolonies and some non-target ground arthropods. Ecotoxicology, 2015; 24: 1221-1228.

8. Zhang B, Xu Z, Zhang Y, Shao X, Xu X, Cheng J, et al. Fipronil induces apoptosis through caspase-dependent mitochondrial pathways in Drosophila S2 cells. Pestic Biochem Physiol, 2015; 119: 81-89.

9. de Medeiros HC, Constantin J, Ishii-Iwamoto EL, Mingatto FE. Effect of fipronil on energy metabolism in the perfused rat liver. Toxicol Lett, 2015; 236: 34-42.

10. Badgujar PC, Pawar NN, Chandratre GA Telang AG, Sharma AK. Fipronil induced oxidative stress in kidney and brain of mice: protective effect of vitamin E and vitamin C. Pestic Biochem Physiol, 2015;118: 10-18.

11. Merkowsky K, Sethi RS, Gill JP, Singh B.
Fipronil induces lung inflammation in vivo and cell death in vitro. J Occup Med Toxicol, 2016; 11: 10.

12. Bonmatin JM, Giorio C, Girolami V, Goulson D, Kreutzweiser DP, Krupke C, et al. Environmental fate and exposure; neonicotinoids and fipronil. Environ Sci Pollut Res Int, 2015; 22: 35-67.

13. Al-Harbi MS. Fipronil Induced Hepatotoxicity, Genotoxicity, Oxidative Stress and the Possible Ameliorative Effect of Ginseng. Br J Pharmaceut Res, 2016; 14.

14. Romero A, Ramos E, Ares I, Castellano V, Martinez M, Martinez-Larranaga MR, et al. Fipronil sulfone induced higher cytotoxicity than fipronil in SH-SY5Y cells: Protection by antioxidants. Toxicol Lett, 2016; 252: 42-49.

15. Rincon M, Irvin CG. Role of IL-6 in asthma and other inflammatory pulmonary diseases. Int J Biol Sci, 2012; 8: 1281-1290.

16. Barnes PJ. The cytokine network in asthma and chronic obstructive pulmonary disease. J Clin Invest, 2008; 118: 3546-3556.

17. Allen TC, Kurdowska A. Interleukin 8 and acute lung injury. Arch Pathol Lab Med, 2014; 138: 266-9.

18. Di Stefano A, Capelli A, Donner CF. Role of interleukin-8 in the pathogenesis and treatment of COPD. Chest, 2004; 126: 676-678.

19. Fahy JV. Goblet cell and mucin gene abnormalities in asthma. Chest, 2002; 122: 320S-326S.

20. Alagha K, Bourdin A, Vernisse C, Garulli C, Tummino C, Charriot J, et al. Goblet cell hyperplasia as a feature of neutrophilic asthma. Clin Exp Allergy, 2019; Epub.

21. Ammit AJ, Panettieri RA, Jr. Airway smooth muscle cell hyperplasia: a therapeutic target in airway remodeling in asthma? Prog Cell Cycle Res, 2003; 5: 49-57.

22. Shaykhiev R. Emerging biology of persistent mucous cell hyperplasia in COPD. Thorax, 2019; 74: 4-6.

23. Rogers DF. Airway goblet cell hyperplasia in asthma: hypersecretory and anti-inflamma- tory? Clin Exp Allergy, 2002; 32: 1124-1127.

24. Kim DH, Chu HS, Lee JY, Hwang SJ, Lee SH, Lee HM. Up-regulation of MUC5AC and MUC5B mucin genes in chronic rhinosinusitis. Arch Otolaryngol Head Neck Surg, 2004 130: 747-752.

25. Roy MG, Livraghi-Butrico A, Fletcher AA, McElwee MM, Evans SE, Boerner RM, et al. Muc5b is required for airway defence. Nature, 2014; 505: 412-416+

26. Ermund $A$, Meiss LN, Rodriguez-Pineiro AM Bahr A, Nilsson HE, Trillo-Muyo S, et al. The normal trachea is cleaned by MUC5B mucin bundles from the submucosal glands coated with the MUC5AC mucin. Biochem Bioph Res Co, 2017; 492: 331-337.

27. Martinez-Anton A, de Bolos C, Alobid I, Benitez P, Roca-Ferrer J, Picado C, et al. Corticosteroid therapy increases membrane-tethered while decreases secreted mucin expression in nasal polyps. Allergy. 2008; 63: 1368-1376.

Yong-Dae Kim, M.D., Ph.D.

Department of Otorhinolaryngology-

Head and Neck Surgery

College of Medicine

Yeungnam University

170 Hyeonchung-ro

Nam-gu, Daegu 42415

Republic of Korea

Tel: $+82-53-620-3781$

Fax: $+82-53-628-7884$

E-mail:ydkim@med.yu.ac.kr 